\title{
Effect of Feline Interferon-Omega on the Survival Time and Quality of Life of Cats with Feline Infectious Peritonitis
}

\author{
Susanne Ritz, Herman Egberink, and Katrin Hartmann
}

\begin{abstract}
Background: There is no therapy with proven efficacy to treat cats with feline infectious peritonitis (FIP).
Hypothesis: Feline interferon-omega (FeIFN- $\omega)$ prolongs survival time and increases quality of life in cats with FIP. Animals: Thirty-seven privately owned cats were subjects of this study.

Methods: The study was performed as a placebo-controlled double-blind trial. Feline infectious peritonitis was confirmed by histology or immunostaining of feline coronavirus (FCoV) antigen in effusion or tissue macrophages or both. The cats were randomly selected for treatment with either FeIFN- $\omega$ or a placebo. All cats received adjunctive treatment with glucocorticoids and antibiotics and passive immunization with Feliserin.

Results: There was no statistically significant difference in the survival time of cats treated with FeIFN- $\omega$ versus placebo or in any other variable evaluated (with the exception of the lymphocyte count). The cats survived between 3 and 200 days (median, 9 days). There was only 1 long-term survivor ( $>3$ months), and the cat was in the FeIFN- $\omega$ group.

Conclusion and Clinical Relevance: No effect of FeIFN- $\omega$ on survival time or quality of life could be demonstrated in this study.

Key words: Feline coronavirus (FCoV); Glucocorticoids; Therapy; Treatment.
\end{abstract}

$\mathbf{F}$ eline infectious peritonitis (FIP) is a common disease of cats that occurs worldwide. ${ }^{1}$ It is an immune-mediated disease that is triggered by infection with a feline coronavirus $(\mathrm{FCoV}){ }^{2} \mathrm{FCoV}$ exists in 2 serotypes (serotype I and II), ${ }^{3}$ which differ in growing behavior and cell cytotoxicity in vitro but not in their potential to cause FIP. ${ }^{4}$ FIP is a deadly disease, and there are no confirmed reports of cats that have recovered from it. Although anecdotal reports state that some cats might survive $2-5$ weeks after onset of clinical signs, ${ }^{5}$ the duration of survival is poorly documented.

Several studies of treatment of cats with FIP have been published. However, only a few had more than 12 cats per group, most studies did not include control groups, and FIP was not reliably confirmed. Recently 12 cats suspected of having FIP were treated with $10^{6} \mathrm{U} / \mathrm{kg}$ feline interferon-omega (FeIFN- $\omega$ ) every other day until remission, followed by weekly injections. Four of the 12 cats lived longer than 2 years. No control group was included in the study.

From the Clinic of Small Animal Internal Medicine, Ludwig Maximilian University Munich, Germany (Ritz, Hartmann); and the Department of Infectious Diseases and Immunology, Division of Virology, Faculty of Veterinary Medicine, Utrecht University, the Netherlands (Egberink). Part of this work previously presented at the German Conference of Veterinary Internal Medicine and Clinical Pathology, Munich, Germany, May 13-14, 2006; at the American College of Veterinary Medicine Forum, Louisville, KY, May 31-June 3, 2006; at the European College of Veterinary Internal Medicine (ECVIM) Congress, Amsterdam, the Netherlands, September 1416, 2006; and at the 8th International Feline Retrovirus Research Symposium, Washington, DC, October 8-11, 2006.

Reprint requests: Katrin Hartmann, Prof, Dr Dr habil, Dip ECVIM-CA, Clinic of Small Animal Medicine, Ludwig Maximilian University Munich, Veterinaerstrasse 13, 80539 Munich, Germany; e-mail: Hartmann@uni-muenchen.de.

Submitted December 14, 2006; Revised March 5, 2007, April 15, 2007; Accepted May 18, 2007.

Copyright $(0) 2007$ by the American College of Veterinary Internal Medicine

0891-6640/07/2106-0005/\$3.00/0
The aim of this study was to evaluate the efficacy of FeIFN- $\omega$ in cats with a confirmed diagnosis of FIP in a placebo-controlled double-blind trial.

\section{Materials and Methods \\ Cats Included}

The study was designed as a randomized double-blind study including 37 cats. Inclusion criterion to enter the study was the definitive diagnosis of FIP. All cats were presented to the Clinic of Small Animal Internal Medicine, Ludwig Maximilian University Munich, Germany. Cats were included in the study from August 2004 to April 2006. An informed consent of participation signed by the owners was obtained for all cats. This study fulfilled the general German guidelines for prospective studies with owners' consents. These cats were consecutive cases with confirmed FIP that had owners willing to participate in the study.

Effusion was present in 36 of the 37 cats (97\%). The diagnosis in these cats was confirmed by detection of FCoV antigen in macrophages in the effusion. ${ }^{6}$ Biopsies of the liver, mesenteric lymph nodes, kidney, spleen, and omentum had typical histologic signs of FIP and positive immunohistochemical staining of FCoV antigen in macrophages in the remaining cat. ${ }^{7}$ Only cats that were feline leukemia virus-(FeLV) and feline immunodeficiency virus (FIV)-negative on Speed DUO FeLV-FIV test ${ }^{\mathrm{a}}$ were included. Cats were excluded if they survived fewer than 72 hours after treatment initiation.

\section{Study Design}

The study was performed as a placebo-controlled, double-blind study. A follow-up period of 1 year was planned. Cats were randomly assigned to 1 of 2 study groups with a table of random numbers. FeIFN- $\omega^{\mathrm{b}}$ dosage was based on a previous study. ${ }^{8}$ Cats of the interferon group (21 cats) received $10^{6} \mathrm{U} / \mathrm{kg}(0.1 \mathrm{~mL} / \mathrm{kg})$ FeIFN- $\omega$ subcutaneously every 24 hours for 8 days. After day 8 , cats received $10^{6} \mathrm{U} / \mathrm{kg}$ FeIFN- $\omega$ once a week for a total of 1 year or until euthanasia. Cats of the placebo group (16 cats) received 0.1$\mathrm{mL}$ physiologic saline as a placebo at the same schedule. Both substances were coded and could not be distinguished either by the clinicians or technicians treating the cats in the teaching hospital or by the owners. The code was broken after all cats had been treated and all results had been obtained.

All cats were treated with glucocorticoids. In case of effusion, $1-\mathrm{mg} / \mathrm{kg}$ dexamethasone $\mathrm{c}^{\mathrm{c}}$ was administered intraperitoneally or 
intrathoracically every 24 hours as long as effusion was present but no longer than 7 days. After effusion vanished or at day $8,2 \mathrm{mg} / \mathrm{kg}$ prednisolone $^{\mathrm{d}, \mathrm{e}}$ was administered PO every 24 hours. Cats without effusion received $2 \mathrm{mg} / \mathrm{kg}$ prednisolone every 24 hours from the very beginning. Additional treatment of all cats included amoxicillin/clavulanic acid $(12.5 \mathrm{mg} / \mathrm{kg}$ IV every 12 hours for 8 days), dalteparin-natrium ${ }^{\mathrm{g}}$ (75 IU/kg subcutaneously every 12 hours for 5 days, then gradually tapered during next 2 days), and a product (Feliserim) containing antibodies ${ }^{\mathrm{h}}$ against feline parvovirus (FPV), feline herpesvirus (FHV), and feline calicivirus (FCV) $(4 \mathrm{~mL}$ subcutaneously once). These immunoglobulins were given because many cats were not properly vaccinated and because of their increased risk of acquiring an infectious disease due to immune suppression (use of high-dose glucocorticoids) and their higher risk of acquiring an secondary infections during the hospitalization phase. Antibiotics were administered to minimize the risk of bacterial infection because paracentesis was performed daily. Cats additionally received nutrition management and fluid therapy, as needed.

\section{Examination Schedule}

All cats were hospitalized during the first 8 days after treatment initiation. During hospitalization, physical and ultrasound examinations were performed daily. Depending on their health status, cats were returned to their owners after day 8 . The owners were asked to judge their cats every day and to fill in a provided diary recording temperature, respiratory rate, weight, length of sleeping time, eating, playing, and grooming behaviour, as well as any problem noticed by the owners. Follow-up examinations in the teaching hospital were scheduled on days 7, 14, 30, 90, 180, and 360 , including physical examination and ultrasound to detect the presence of effusion.

The general condition was characterized by the Karnofsky's score. The index enables judgment of quality of life and well-being in cats by means of a score from 100\% (absolutely healthy and happy) to $0 \%$ (dead). ${ }^{9}$ A CBC and a small animal biochemistry profile were performed on day 0 (day of starting treatment) as well as on days $7,14,30,90,180$, and 360 .

\section{Data Analysis}

The cats were assigned to 4 groups according to their survival time. Cats were defined as "very short-term survivors," living $<7$ days; "short-term survivors," living $\geq 7$ but $<21$ days; "medium-term survivors," living $\geq 21$ days but $<3$ months; and "longterm survivors," living $\geq 3$ months (Table 1). Statistical analysis

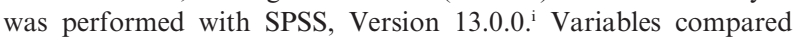
between both groups included Karnofsky's score, erythrocytes, hematocrit, platelets, hemoglobin, leukocytes, monocytes, lymphocytes, banded neutrophils, mature neutrophils, eosinophils, basophils, alanine aminotransferase, alkaline phosphatase, bilirubin, protein, albumin, urea, creatinine, glucose, chloride, and phosphorus. A difference in the survival time between both groups was evaluated with a log-rank test. Differences between the 2 groups at day 0 (to rule out a bias between groups), day 7 , and day 14 were investigated with a Student's $t$-test or a Mann-Whitney $U$-test. The $t$-test was used for normally distributed data; the Mann-Whitney $U$-test was used for all other parameters. $P$ values of $\leq .05$ were considered significant. A Bonferroni correction was performed to rule out multiple test interference. A $5 \%$ significance level was assumed for all variables, and thus, .05 was divided through the number of tests performed (44). Therefore, a final $P \leq .002$ for each variable was considered significant.

\section{Results}

Thirty-two cats $(87 \%)$ were European Shorthair cats, $3(8 \%)$ were British Shorthair cats, and $2(5 \%)$ were Persian cats. Age ranged from 8 weeks to 7 years (mean, 1 year; median, 0.5 years). Twenty $(54 \%)$ cats were younger than 6 months; $34(92 \%)$ cats were younger than 24 months. Twenty-six $(70 \%)$ cats were male (14 neutered), and $11(30 \%)$, female (3 neutered). Of the 36 cats that presented with effusion, $31(86 \%)$ had ascites, 2 $(6 \%)$ had ascites and scrotal effusion, $2(6 \%)$ had thoracic effusion, and 1 cat $(3 \%)$ had ascites as well as thoracic effusion. Twenty cats were randomly assigned to the FeIFN- $\omega$ group, and 17 cats to the placebo group.

There was no statistically significant difference in any variable on day 0 between cats later treated with FeIFN$\omega$ and those that later received placebo (Table 2). There was a significant difference $(P \leq .001)$ between the lymphocyte counts (reference interval $1-4 \times 10^{9} / \mathrm{L}$ ) of FeIFN- $\omega$-treated versus cats receiving placebo on day 7 . In the FeIFN- $\omega$ group, the mean lymphocyte count was $0.5 \pm 0.7 \times 10^{9} / \mathrm{L}$; in the placebo group, the mean lymphocyte count was $1.7 \pm 2.1 \times 10^{9} / \mathrm{L}$. There was no statistically significant difference in the Karnofsky's score of both groups on any of the days evaluated (day 0 to day 14) (Fig 1). No statistical evaluation was performed after day 14 because only 5 cats remained in the study.

Cats survived between 3 and 200 days (median, 9 days). The median survival time of cats belonging to the FeIFN- $\omega$ group was 9 days. Median survival time of the placebo group was 8 days. The difference in survival time between both groups was not significant (log-rank test, $P=.6290)$ (Fig 2). Thirty-two of $37(87 \%)$ of cats survived fewer than 4 weeks. Cats were classified in 4 groups according to their survival time (Table 1). Seven of the 37 cats improved in their general condition during treatment ( 2 cats receiving FeIFN- $\omega, 5$ cats receiving placebo). Five cats (the long-term survivor and 4 medium-term survivors) developed a secondary infection (upper respiratory tract disease, panleukopenia, or bacterial cystitis) during treatment (after 9, 25, 51, and

Table 1. Classification of the cats in 4 groups according to their survival time.

\begin{tabular}{|c|c|c|c|c|}
\hline & Very Short-Term Survivors & Short-Term Survivors & Medium-Term Survivors & Long-Term Survivors \\
\hline Cats & $(<7 \mathrm{~d})$ & $(\geq 7$ to $<21 \mathrm{~d})$ & $(\geq 21$ to $<3 \mathrm{~d})$ & $(\geq 3 \mathrm{mo})$ \\
\hline FeIFN- $\omega$ group & $8(8 / 21)$ & $10(10 / 21)$ & $2(2 / 21)$ & $1(1 / 21)$ \\
\hline Placebo group & $8(8 / 16)$ & $4(4 / 16)$ & $4(4 / 16)$ & $0(0 / 16)$ \\
\hline All cats & $16(16 / 37)$ & $14(14 / 37)$ & $6(6 / 37)$ & $1(1 / 37)$ \\
\hline
\end{tabular}

FeIFN- $\omega$, feline interferon-omega. 
Table 2. Variables on day 0 and $P$ value of all parameters showing (bold) or denying a significant difference between the FeIFN- $\omega$ and the placebo group on days 7 and 14.

\begin{tabular}{|c|c|c|c|c|c|}
\hline \multirow[b]{3}{*}{ Parameter } & \multirow[b]{3}{*}{ Unit } & \multirow{2}{*}{$\frac{\text { Day } 0}{\text { FeIFN- } \omega \text { Group }}$} & \multirow{2}{*}{$\frac{\text { Day } 0}{\text { Placebo Group }}$} & \multirow{2}{*}{$\frac{\text { Day } 7}{\text { FeIFN- } \omega \text { vs Placebo }}$} & \multirow{2}{*}{$\frac{\text { Day } 14}{\text { FeIFN- } \omega \text { vs Placebo }}$} \\
\hline & & & & & \\
\hline & & $($ mean $\pm \mathrm{SD})$ & $($ mean $\pm \mathrm{SD})$ & $(P$ value $)$ & $(P$ value $)$ \\
\hline Karnofsky & $\%$ & $73 \pm 10$ & $76 \pm 12$ & .443 & .393 \\
\hline Erythrocytes & $\times 10^{12} / \mathrm{L}$ & $7.70 \pm 2.58$ & $6.98 \pm 1.15$ & .896 & .662 \\
\hline Hematocrit & $\mathrm{L} / \mathrm{L}$ & $0.319 \pm 0.101$ & $0.279 \pm 0.068$ & .227 & .705 \\
\hline Platelets & $\times 10^{9} / \mathrm{L}$ & $218.0 \pm 113.9$ & $307.0 \pm 218.4$ & .120 & .519 \\
\hline Hemoglobin & $\mathrm{mM}$ & $6.45 \pm 2.04$ & $5.90 \pm 1.10$ & .663 & .317 \\
\hline Leukocytes & $\times 10^{9} / \mathrm{L}$ & $18.56 \pm 9.33$ & $16.20 \pm 10.25$ & .664 & .024 \\
\hline Monocytes & $\times 10^{9} / \mathrm{L}$ & $0.31 \pm 0.45$ & $0.25 \pm 0.42$ & .705 & .452 \\
\hline Lymphocytes & $\times 10^{9} / \mathrm{L}$ & $1.24 \pm 1.70$ & $1.45 \pm 1.23$ & .001 & .143 \\
\hline Band neutrophils & $\times 10^{9} / \mathrm{L}$ & $0.94 \pm 1.36$ & $0.53 \pm 1.14$ & .554 & .786 \\
\hline Mature neutrophils & $\times 10^{9} / \mathrm{L}$ & $16.0 \pm 8.44$ & $13.35 \pm 9.16$ & .808 & .036 \\
\hline Eosinophils & $\times 10^{9} / \mathrm{L}$ & $0.05 \pm 0.12$ & $0.11 \pm 0.30$ & .393 & .714 \\
\hline Basophils & $\times 10^{9} / \mathrm{L}$ & $0.00 \pm 0.00$ & $0.00 \pm 0.00$ & .695 & 1.000 \\
\hline ALT & $\mathrm{U} / \mathrm{L}$ & $73.3 \pm 74.9$ & $42.4 \pm 22.2$ & .941 & .067 \\
\hline ALP & $\mathrm{U} / \mathrm{L}$ & $28.0 \pm 24.3$ & $20.0 \pm 18.1$ & 1.000 & .297 \\
\hline Bilirubin & $\mu \mathrm{M}$ & $29.3 \pm 32.1$ & $32.3 \pm 40.5$ & .051 & .879 \\
\hline Protein & $\mathrm{g} / \mathrm{L}$ & $77.1 \pm 16.4$ & $73.5 \pm 19.7$ & .843 & 1.000 \\
\hline Albumin & $\mathrm{g} / \mathrm{L}$ & $22.4 \pm 3.9$ & $23.0 \pm 4.5$ & .317 & 1.000 \\
\hline Urea & $\mathrm{mM}$ & $6.91 \pm 2.14$ & $6.62 \pm 1.90$ & .370 & .037 \\
\hline Creatinine & $\mu \mathrm{M}$ & $60.2 \pm 23.3$ & $57.8 \pm 25.0$ & .341 & .620 \\
\hline Glucose & $\mathrm{mM}$ & $7.19 \pm 2.45$ & $5.76 \pm 1.50$ & .766 & .074 \\
\hline Chloride & $\mathrm{mM}$ & $116 \pm 1$ & $116 \pm 8$ & .175 & .629 \\
\hline Phosphorus & $\mathrm{mM}$ & $2.01 \pm 0.37$ & $1.90 \pm 0.41$ & .295 & .110 \\
\hline
\end{tabular}

FeIFN- $\omega$, feline interferon-omega; Karnofsky, Karnofsky's score; ALT, alanine aminotransferase; ALP, alkaline phosphatase.

180 days, respectively), after which they deteriorated rapidly and died or had to be euthanized after 22, 26, 53, and 200 days, respectively. The effusion resolved in 6 cats during treatment ( 3 cats receiving FeIFN- $\omega, 3$ cats

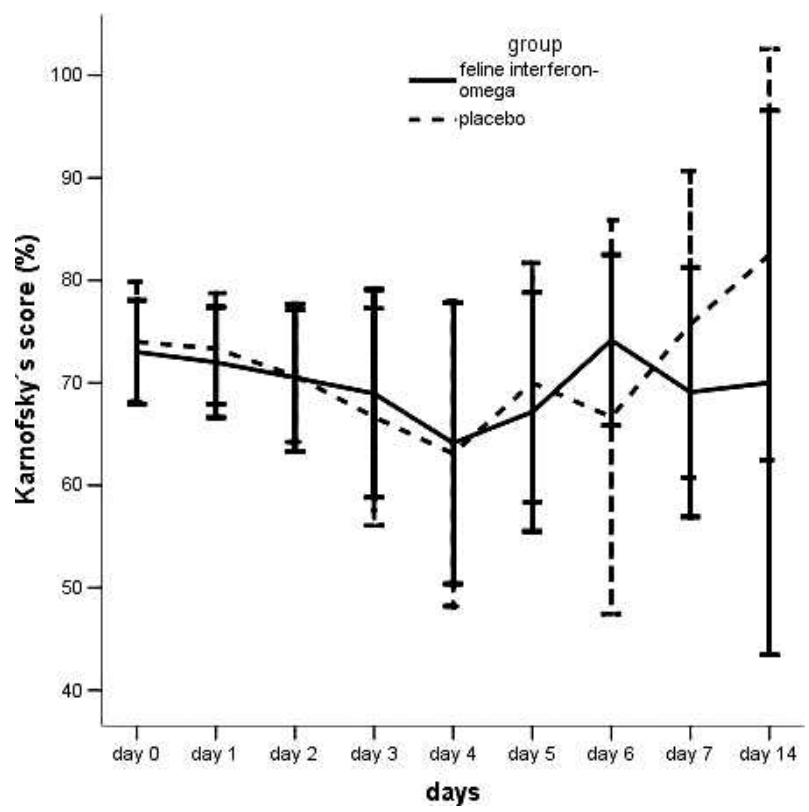

Fig 1. Karnofsky's scores, an indicator of quality of life, of cats treated with feline interferon-omega and of cats treated with placebo from day 0 to day 14 . The error bars have a coincidence interval of $95 \%$, and the other bar represents the median. receiving placebo). Of these 6 cats, the long-term survivor, 1 medium-term survivor, and 1 very shortterm survivor received FeIFN- $\omega$. The other 3 cats in which the effusion resolved received placebo ( 3 mediumterm survivors). Effusion relapsed in all 6 cats a few days before they were euthanized.

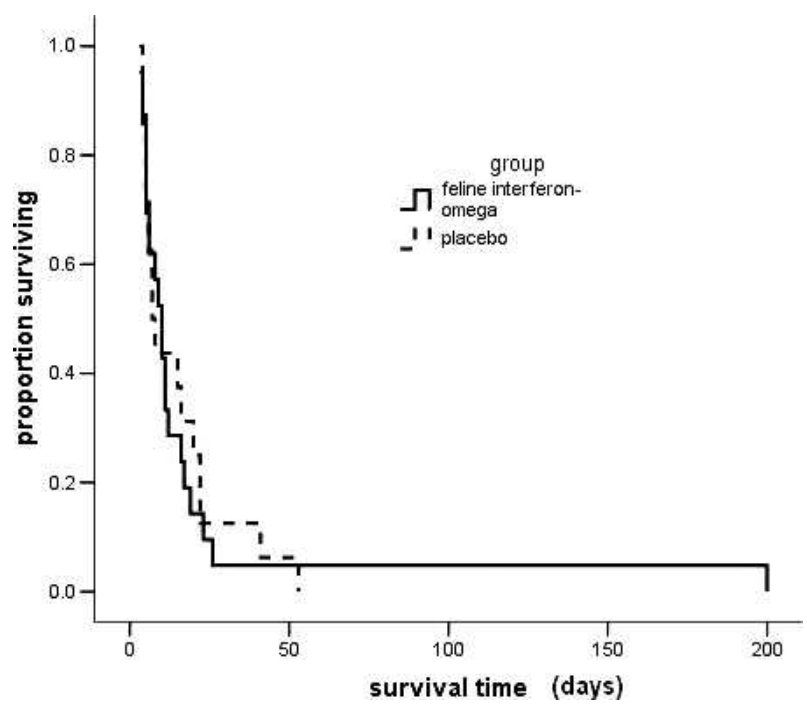

Fig 2. Kaplan-Meier survival curve of cats treated with feline interferon-omega and cats treated with a placebo. 


\section{Discussion}

Nearly all cats $(36 / 37,97 \%)$ in this study presented with effusion. In other studies, between 60 and $84 \%$ of the cats with FIP had effusions., ${ }^{6,10,11}$ It is far more difficult to diagnose FIP if no effusion is present. More invasive techniques (biopsies in laparoscopy or laparotomy) are required to confirm FIP in such cases. Therefore, it is likely that the proportion of cats with effusive FIP might be over-represented in the present study because only cats with confirmed diagnosis were enrolled.

There are only a few reports on survival times of cats with FIP, most of them being only anecdotal reports without clearly defining survival time after definitive diagnosis of FIP. ${ }^{5,12}$ Thus, a comparison of the survival time in the present study with published data is difficult. There was no significant difference in the survival time or the Karnofsky's scores of cats treated with FeIFN- $\omega$ versus cats treated with placebo. A difference between groups was found only in that cats treated with FeIFN$\omega$ had a significantly lower lymphocyte count. The most likely explanation is a direct effect of the FeIFN- $\omega$ on the lymphocyte count.

The reasons for the lack of efficacy of FeIFN- $\omega$ compared with placebo are uncertain. The FeIFN- $\omega$ might not reach tissue concentrations sufficient to exhibit an antiviral effect. FeIFN- $\omega$ could not be found in the brain, and only traces could be detected in muscle tissue. ${ }^{13}$ Thus, FeIFN- $\omega$ might not have reached the necessary concentration in all tissues to affect $\mathrm{FCoV}$ replication in the cats of the present study. In addition, FeIFN- $\omega$ has a half-life of $31 \pm 5$ minutes, and treatment intervals in the present study might have been too long. However, its action is much longer than its half-life because FeIFN- $\omega$ activates the cellular enzyme $2^{\prime}, 5^{\prime}$-oligoadenylate synthetase, which stays active for more than 3 days after induction. This enzyme is mainly responsible for the antiviral action. ${ }^{13}$

Alternatively, treatment might have been initiated too late. If immune-mediated processes in FIP become autonomous and proceed independently of the presence of the virus, a reduction of the virus load through FeIFN- $\omega$ might not have any appreciable effect. Signs of FIP become apparent 1-2 weeks after experimental inoculation of mutated FCoV. ${ }^{14}$ Ideally, therapy should start at the time of the mutation, before the mutated virus replicates and invades different tissues. The time point of mutation, however, cannot be detected because mutation precedes clinical signs, and a diagnosis is not possible until severe immune-mediated reactions are present.

Previously 4 of 12 cats treated with FeIFN- $\omega$ survived longer than 2 years. ${ }^{6}$ However, in this current study the longest survival time was 200 days in 1 single cat. There are 3 potential reasons why the results of the present study are different from those in the study of Ishida et al (2004). ${ }^{8}$ The first reason for the discrepancy could be the predominance of different serotypes of FCoV (serotype I versus serotype II). ${ }^{3}$ In Japan, $30 \%$ of cats having FIP are infected with serotype II,${ }^{15}$ whereas in Europe, only
$14 \%$ harbor serotype II. ${ }^{16}$ The prognosis might be better in cats infected with serotype II.

A second possible reason could be the additional drugs used in the study. Cats received an immunosuppressive dose of glucocorticoids $(2 \mathrm{mg} / \mathrm{kg}$ ) in the present study, which seemed to promote secondary infections in 5 cats. These cats had improved, and other infections preceded the recurrence of the signs of FIP. Potentially, a slow tapering of the glucocorticoids might be better in cats living longer than 3 weeks to reduce the risk of secondary infections. In the present study, cats received amoxicillin/clavulanic acid and passive immunization in addition. Amoxicillin/clavulanic acid was chosen because of its broad antimicrobial spectrum and because of its few adverse effects, especially in young cats. However, amoxicillin/clavulanic acid is reported to inhibit the interferon-gamma (interferon- $\gamma$ ) activity in human lung epithelia cell lines in vitro, ${ }^{17}$ but no effect could be detected in an in vivo study in humans. ${ }^{18}$ Thus, it might have suppressed endogenous interferon- $\gamma$ in the cats, which is reported to play an important role in the battle against the virus. ${ }^{19}$ Potentially, it also might have decreased the effect of the FeIFN- $\omega$. The immune serum used as adjunctive therapy reduces the risk for virus infections. ${ }^{20}$ No adverse effects of the immune serum are known, and thus, it is unlikely that its application had influence on the outcome of the study.

The third reason explaining the different results could be the fact that the cats in the study of Ishida et al $(2004)^{8}$ had other diseases and not FIP. FIP was not confirmed in any of the 4 cats living at least 2 years in their study.

There are a few studies that used human interferonalpha (interferon- $\alpha$ ) to treat cats with FIP. In vitro, the antiviral efficacy of human interferon- $\alpha$ against a FIPcausing FCoV strain was demonstrated. ${ }^{21}$ Bölcskei and Bilkei (1995) ${ }^{22}$ treated 29 cats suspected of having FIP with different drugs (in 5 groups), and 1 group received human interferon- $\alpha\left(6 \times 10^{5}\right.$ U/cat 5 days a week for 3 weeks), but in this study, FIP was not confirmed but only suspected. Weiss et al $(1990)^{14}$ did a study in 74 specific pathogen-free cats (52 treated, 22 controls) in which FIP was induced experimentally. ${ }^{14}$ Neither the prophylactic nor the therapeutic administration of high doses of interferon- $\alpha\left(10^{4} \mathrm{U} / \mathrm{kg}\right.$ or $\left.10^{6} \mathrm{U} / \mathrm{kg}\right)$ or feline interferon-beta $\left(10^{3} \mathrm{U} / \mathrm{kg}\right)$ significantly reduced mortality in treated versus untreated cats. However, in cats treated with $10^{6} \mathrm{U} / \mathrm{kg}$ interferon- $\alpha$, the survival time was significantly increased (from 5 days in untreated cats to 14 days in treated cats) when treatment was started before virus inoculation. The major difference between the study of Weiss et al (1990) ${ }^{14}$ and the present study is the time point of treatment initiation. Weiss et al (1990) ${ }^{14}$ started the human interferon$\alpha$ treatment before virus was inoculated, whereas the FeIFN- $\omega$ treatment in the present study was started after FIP had developed. FeIFN- $\omega$ also might be more effective if started earlier, but this is not of relevance in the treatment of cats with FIP in the field because a diagnosis needs to be obtained before treatment is started. 


\section{Footnotes}

${ }^{a}$ Bio Veto Test-Diagnostic Veterinaire, La Seyne-sur-mer, France

${ }^{\mathrm{b}}$ Virbagen Omega, Virbac SA, Carros, France

${ }^{\mathrm{c}}$ Hexadreson, Intervet, Unterschleißheim, Germany

${ }^{\mathrm{d}}$ Prednisolon, 2 mg, GALENpharma GmbH, Kiel, Germany

${ }^{\mathrm{e}}$ Prednisolon, $5 \mathrm{mg}$, CP-Pharma, Burgdorf, Germany

${ }^{\mathrm{f}}$ Augmentan, GlaxoSmithKline GmbH \& Co KG, Munich, Germany

${ }^{g}$ Fragmin, Pfizer Pharma GmbH, Karlsruhe, Germany

${ }^{\mathrm{h}}$ FELISERIN, Impfstoffwerk Dessau-Tornau GmbH, Rodleben, Germany

${ }^{i}$ SPSS Inc, Chicago, IL

\section{Acknowledgments}

We thank Karine de Mari, of Virbac SA, France, for support in this study and the Department of Statistics, Ludwig Maximilian University Munich, Germany, for their help with the statistical evaluation.

\section{References}

1. Rohrbach BW, Legendre AM, Baldwin CA, et al. Epidemiology of feline infectious peritonitis among cats examined at veterinary medical teaching hospitals. J Am Vet Med Assoc 2001;218:1111-1115.

2. Pedersen NC. Virologic and immunologic aspects of feline infectious peritonitis virus infection. Adv Exp Med Biol 1987;218:529-550.

3. Pedersen NC, Black JW, Boyle JF, et al. Pathogenic differences between various feline coronavirus isolates. Adv Exp Med Biol 1984;173:365-380.

4. Herrewegh AA, Smeenk I, Horzinek MC, et al. Feline coronavirus type II strains 79-1683 and 79-1146 originate from a double recombination between feline coronavirus type I and canine coronavirus. J Virol 1998;72:4508-4514.

5. Robison RL, Holzworth J, Gilmore CE. Naturally occurring feline infectious peritonitis: Signs and clinical diagnosis. J Am Vet Med Assoc 1971;158:981-986.

6. Hartmann K, Binder C, Hirschberger J, et al. Comparison of different tests to diagnose feline infectious peritonitis. J Vet Intern Med 2003;17:781-790.

7. Tammer R, Evensen O, Lutz H, et al. Immunohistological demonstration of feline infectious peritonitis virus antigen in paraffin-embedded tissues using feline ascites or murine monoclonal antibodies. Vet Immunol Immunopathol 1995;49:177-182.

8. Ishida T, Shibanai A, Tanaka S, et al. Use of recombinant feline interferon and glucocorticoid in the treatment of feline infectious peritonitis. J Feline Med Surg 2004;6:107-109.

9. Hartmann K, Kuffer M. Karnofsky's score modified for cats. Eur J Med Res 1998;3:95-98.

10. Walter JH, Rudolph R. The frequency and pathogenesis of feline infectious peritonitis (FIP) (in German). Dtsch Tierarztl Wochenschr 1989;96:194-196, 201.

11. Lutz H, Hauser B, Horzinek MC. Feline infectious peritonitis (FIP): The present state of knowledge. J Small Anim Pract 1986;27:108-116.

12. McReynolds C, Macy D. Feline infectious peritonitis. Part I. Etiology and diagnosis. Comp Cont Educ Pract Vet 1997; 19:1007-1016.

13. Ueda Y, Sakurai T, Kasama K, et al. Pharmacokinetic properties of recombinant feline interferon and its stimulatory effect on $2^{\prime}, 5^{\prime}$-oligoadenylate synthetase activity in the cat. J Vet Med Sci 1993;55:1-6.

14. Weiss RC, Cox NR, Oostrom-Ram T. Effect of interferon or Propionibacterium acnes on the course of experimentally induced feline infectious peritonitis in specific-pathogen-free and random-source cats. Am J Vet Res 1990;51:726-733.

15. Hohdatsu T, Okada S, Ishizuka $\mathrm{Y}$, et al. The prevalence of types I and II feline coronavirus infections in cats. J Vet Med Sci 1992;54:557-562.

16. Benetka V, Kubber-Heiss A, Kolodziejek J, et al. Prevalence of feline coronavirus types I and II in cats with histopathologically verified feline infectious peritonitis. Vet Microbiol 2004;99:31-42.

17. Brooks BM, Hart CA, Coleman JW. Differential effects of $\beta$-lactams on human IFN- $\gamma$ activity. J Antimicrob Chemother 2005;56:1122-1125.

18. Dufour V, Millon L, Faucher J-F, et al. Effects of a shortcourse of amoxicillin/clavulanic acid on systemic and mucosal immunity in healthy adult humans. Int Immunopharmacol 2005; 5:917-928.

19. Kyuwa S, Tagawa $\mathrm{Y}$, Machii $\mathrm{K}$, et al. MHV-induced fatal peritonitis in mice lacking IFN-gamma. Adv Exp Med Biol 1998;440:445-450.

20. Bertens AP. Passive and active immunization against feline enteritis and rhinotracheitis in experimental cats (in Dutch). Tijdschr Diergeneeskd 1979;104:107-110.

21. Weiss RC, Oostrom-Ram T. Inhibitory effects of ribavirin alone or combined with human alpha interferon on feline infectious peritonitis virus replication in vitro. Vet Microbiol 1989;20: 255-265.

22. Bölcskei A, Bilkei G. Langzeitstudie über behandelte FIPverdächtige Katzen. Tierärztl Umschau 1995;50:721-728. 